\title{
Positivstellensatz for homogeneous semialgebraic sets
}

\author{
Aleksandra Gala-Jaskórzyńska, Krzysztof Kurdyka, \\ Katarzyna Kuta, and Staniseaw Spodzieja
}

\begin{abstract}
We call a closed basic semialgebraic set $X \subset \mathbb{R}^{n}$ homogeneous if it is defined by a finite system of inequalities of the form $g(x) \geq 0$, where $g$ is a homogeneous polynomial. We prove an effective version of the Putinar and Vasilescu Positivstellensatz for positive homogeneous polynomials on homogeneous semialgebraic sets.
\end{abstract}

Mathematics Subject Classification. Primary 11E25, 12D15; Secondary $26 \mathrm{~B} 25$.

Keywords. Polynomial, Sum of squares, Semialgebraic set, Positivstellensatz.

1. Introduction. We denote by $\mathbb{R}[x]$ the ring of real polynomials in $x=\left(x_{1}\right.$, $\left.\ldots, x_{n}\right)$ and by $\sum \mathbb{R}[x]^{2}$ the set of sums of squares of polynomials from $\mathbb{R}[x]$. A homogeneous polynomial $f$ is called positive definite if $f(x)>0$ for $x \in$ $\mathbb{R}^{n} \backslash\{0\}$.

An important result concerning nonnegative polynomials is the solution of Hilbert's 17th problem by Artin [1], which states that if a polynomial $f$ is nonnegative on $\mathbb{R}^{n}$, then $f$ is a sum of squares of rational functions.

For positive definite homogeneous polynomials Reznick proved the following theorem (see [10, Theorem 3.12]). Let us start with some notation. We denote by $Q_{n, k}$ the set of finite sums of $k$ th powers of linear functions. Let $|x|=\sqrt{x_{1}^{2}+\cdots+x_{n}^{2}}$. Set

$$
G_{n}^{r}=|x|^{2 r}, \quad r \in \mathbb{N} .
$$

This research was partially supported by OPUS Grant No 2012/07/B/ST1/03293 (Poland), SONATA Grant No 2013/09/D/ST1/03701 (Poland) and ANR grant STAAVF (France). 
Let $p \in \mathbb{R}[x]$ be a positive definite homogeneous polynomial. Set

$$
\epsilon(p)=\frac{\inf \{p(u): u \in S\}}{\sup \{p(u): u \in S\}},
$$

where $S=\left\{x \in \mathbb{R}^{n}:|x|=1\right\}$ is the unit sphere.

Theorem 1 (Reznick). Let $p \in \mathbb{R}[x]$ be a positive definite homogeneous polynomial of degree $d$. Then for any $r \in \mathbb{Z}$ such that

$$
r \geq \frac{n d(d-1)}{(4 \log 2) \epsilon(p)}-\frac{n+d}{2}
$$

we have $p G_{n}^{r} \in Q_{n, d+2 r}$.

Scheiderer [11, Remark 4.6] gave a generalization of Reznick's theorem by showing that $|x|^{2}$ in the definition of $G_{n}^{r}$ can be replaced by any positive definite form. Interesting contributions in this context are also [13, Theorem $5.1]$ and [15].

In real algebraic geometry, important problems concern nonnegative polynomials on closed semialgebraic sets. The deepest result in this topic is the Krivine Positivstellensatz [4] rediscovered by Stengle [16]. It states that if

$$
X=\left\{x \in \mathbb{R}^{n}: g_{1}(x) \geq 0, \ldots, g_{r}(x) \geq 0\right\},
$$

where $g_{1}, \ldots, g_{r} \in \mathbb{R}[x]$, then every polynomial $f$ that is strictly positive on $X$ is of the form $h_{1} f=1+h_{2}$ for some polynomials $h_{1}, h_{2}$ from the preordering

$$
\begin{aligned}
& T\left(g_{1}, \ldots, g_{r}\right) \\
& \quad:=\left\{\sum_{e=\left(e_{1}, \ldots, e_{r}\right) \in\{0,1\}^{r}} \sigma_{e} g_{1}^{e_{1}} \cdots g_{r}^{e_{r}}: \sigma_{e} \in \sum \mathbb{R}[x]^{2} \text { for } e \in\{0,1\}^{r}\right\} .
\end{aligned}
$$

In the case of compact basic semialgebraic sets, an important result was obtained by Schmüdgen (see [12]): if the set $X$ is compact, then every polynomial $f$ that is strictly positive on $X$ belongs to the preordering $T\left(g_{1}, \ldots, g_{r}\right)$. Moreover, as proved by Putinar [7], under some additional assumption, $f$ belongs to the quadratic module

$$
P\left(g_{1}, \ldots, g_{r}\right):=\left\{\sigma_{0}+\sigma_{1} g_{1}+\cdots+\sigma_{r} g_{r}: \sigma_{i} \in \sum \mathbb{R}[x]^{2}, i=0, \ldots, r\right\} .
$$

Jacobi found an algebraic proof of this fact [2, Theorem 7] (see also [3]). A version of the above results was obtained in [5]: if $X$ is compact, $f$ is strictly positive on $X$, and $g(x):=R^{2}-|x|^{2} \geq 0$ on $X$ for some $R>0$, then $f$ belongs to

$$
\mathcal{K}\left(g, g_{1}, \ldots, g_{r}\right):=T(g)+\left\{\varphi\left(g_{1}\right) g_{1}+\cdots+\varphi\left(g_{r}\right) g_{r}: \varphi \in \sum \mathbb{R}[t]^{2}\right\} .
$$

In this context for every closed basic semialgebraic set Putinar and Vasilescu proved the following Positivstellensatz (see [9, Theorem 4.2], [8]). 
Theorem 2 (Putinar, Vasilescu). Let $\left(p_{1}, \ldots, p_{m}\right)$ be an m-tuple of real polynomials in $t \in \mathbb{R}^{n}$, and let

$$
\phi(t)=\left(1+|t|^{2}\right)^{-1}, \quad t \in \mathbb{R}^{n} .
$$

Let $p$ be a real polynomial on $\mathbb{R}^{n}$. Suppose that the degrees of $p_{j}$ 's and $p$ are all even.

Let $P_{1}, \ldots, P_{m}, P$ be the homogenizations of the polynomials $p_{1}, \ldots, p_{m}, p$ respectively and assume that $P(x)>0$ whenever $x \in\left\{x \in \mathbb{R}^{n+1}: P_{i}(x) \geq\right.$ $0, i=1, \ldots, m\}, x \neq 0$.

Then there exist an integer $b \geq 0$ and a finite collection of real polynomials $q_{l}, q_{k l}, l \in L, k=1, \ldots, m$, such that

$$
p(t)=\phi(t)^{2 b}\left(\sum_{l \in L} q_{l}(t)^{2}+\sum_{k=1}^{m} \sum_{l \in L} p_{k}(t) q_{k l}(t)^{2}\right), \quad t \in \mathbb{R}^{n} .
$$

The aim of this article is to simplify the representation (1) for any homogeneous polynomial $p$. We will show

Theorem 3. Let $f \in \mathbb{R}[x]$ be a homogeneous polynomial of positive even degree $d$, and let $g_{1}, \ldots, g_{r} \in \mathbb{R}[x]$ be homogeneous polynomials of even degrees. Set

$$
X=\left\{x \in \mathbb{R}^{n}: g_{1}(x) \geq 0, \ldots, g_{r}(x) \geq 0\right\} .
$$

If $f(x)>0$ for $x \in X \backslash\{0\}$, then there exist positive even integers $D, N, a$ polynomial $q \in Q_{n, D}$, and $a, b \in \mathbb{R}$ such that

$$
f(x)=|x|^{-D+d}\left(q+\sum_{i=1}^{r}|x|^{\alpha_{i}}\left(a g_{i}(x)+b|x|^{\operatorname{deg} g_{i}}\right)^{N} g_{i}(x)\right),
$$

where $\alpha_{i}=D-(N+1) \operatorname{deg} g_{i}$ for $i=1, \ldots, r$ are nonnegative even numbers.

In the proof of Theorem 3, we will use the method from [5] and apply the Reznick theorem.

From Theorem 3 we immediately obtain a version of the Putinar-Vasilescu theorem.

Corollary 1. Under the assumptions and notation of Theorem 2, there are even integers $b, D, N \geq 0$ such that $D-(N+1) \operatorname{deg} p_{k} \geq 0$ for $k=1, \ldots, r$, and $a$ finite collection of real polynomials $q_{l}, l \in L$, with $\operatorname{deg} q_{l} \leq 1$ and polynomials $q_{k, 1}, k=1, \ldots, r$, of the form

$$
q_{k, 1}(t)=\left(1+|t|^{2}\right)^{\alpha_{k}}\left(\xi p_{k}(t)+\eta\left(1+|t|^{2}\right)^{\frac{\operatorname{deg} p_{k}}{2}}\right)^{N}
$$

for some $\xi, \eta \in \mathbb{R}$, where $\alpha_{k}=\frac{D-(N+1) \operatorname{deg} p_{k}}{2}$ for $k=1, \ldots, r$, such that

$$
p(t)=\phi(t)^{b}\left(\sum_{l \in L} q_{l}^{D}(t)+\sum_{k=1}^{m} p_{k}(t) q_{k, 1}\right), \quad t \in \mathbb{R}^{n} .
$$

Theorem 3 and Corollary 1 provide an additional information about how the polynomials defining the basic closed semialgebraic set $X$ are involved in the representation of $f$ and $p$ respectively (comparable to Schweighifer result [14, Lemma 8] and [5]). 
2. Proof of Theorem 3. Let $f, g_{1}, \ldots, g_{r} \in \mathbb{R}[x]$ be homogeneous polynomials of even degrees, and let $X \subset \mathbb{R}^{n}$ be of the form (2).

Lemma 1. There exists a polynomial $\varphi \in \sum \mathbb{R}[t]^{2}$ of the form $\varphi(t)=(a t+b)^{N}$, where $t$ is a single variable and $N$ is an even nonnegative integer, such that

$$
f(x)-\sum_{i=1}^{r} g_{i}(x) \varphi\left(g_{i}(x)\right)>0 \text { for } x \in S .
$$

Proof. In the proof we will use the method of Kurdyka and Spodzieja from [5, Lemma 1].

Let $M>1$ and $A \geq 1$ be constants such that

$$
f(x) \geq-M \quad \text { for } x \in S
$$

and

$$
\left|g_{i}(x)\right| \leq A \quad \text { for } \quad x \in S, \quad i=1, \ldots, r
$$

Let

$$
G_{1}:=\{x \in S: f(x)>0\} .
$$

Then there exists $\eta>0$ such that

$$
G_{2}:=\{x \in S: \operatorname{dist}(x, X) \leq \eta\} \subset G_{1}
$$

and

$$
m:=\min \left\{f(x): x \in G_{2}\right\}>0 .
$$

Since $X \cap S=\left\{x \in S: g_{1}(x) \geq 0, \ldots, g_{r}(x) \geq 0\right\}$ and $S$ is compact, there exists $0<\delta \leq 1$ such that

$$
G_{3}:=\left\{x \in S: g_{i}(x) \geq-\delta \text { for } i=1, \ldots, r\right\} \subset G_{2} .
$$

Take

$$
\varepsilon:=\frac{m}{(r+1) A}, \quad B:=A \frac{M+r \varepsilon}{\delta} .
$$

Then there exist $a, b \in \mathbb{R}, N \in \mathbb{N}$, and a polynomial $\varphi \in \mathbb{R}[t]$ of the form $\varphi(t)=(a t+b)^{N}$, where $N$ is an even nonnegative integer, such that

$$
\begin{array}{ll}
\varphi(t)>B & \text { for } t \in[-A,-\delta], \\
\varphi(t)<\varepsilon & \text { for } t \in[0, A] .
\end{array}
$$

We prove (4). Let $x \in X \cap S$. Then $g_{i}(x) \geq 0$ for $i=1, \ldots, r$. Since $\varphi(t)<\varepsilon$ for $t \in[0, A]$, by $(6)$ we have

$$
g_{i}(x) \cdot \varphi\left(g_{i}(x)\right) \leq A \varepsilon<\frac{m}{r+1} \text { for } i=1, \ldots, r .
$$

Hence, by (7),

$$
f(x)-\sum_{i=1}^{r} g_{i}(x) \cdot \varphi\left(g_{i}(x)\right)>m-r \frac{m}{r+1}>0,
$$

and the assertion (4) holds for $x \in X \cap S$. 
Suppose now that $x \in G_{3} \backslash X$. We may assume that

$$
g_{1}(x), \ldots, g_{k}(x) \geq 0 \text { and } g_{k+1}(x), \ldots, g_{r}(x)<0
$$

for some $0 \leq k<r$. Since $\varphi(t)<\varepsilon$ for $t \in[0, A]$, we have

$$
g_{i}(x) \cdot \varphi\left(g_{i}(x)\right) \leq A \varepsilon<\frac{m}{r} \quad \text { for } \quad i=1, \ldots, k .
$$

Moreover from $\varphi(t) \geq 0$ for $t \in \mathbb{R}$, we have

$$
g_{i}(x) \cdot \varphi\left(g_{i}(x)\right)<0 \text { for } i=k+1, \ldots, r .
$$

Therefore, $f(x)-\sum_{i=1}^{r} g_{i}(x) \cdot \varphi\left(g_{i}(x)\right)>m-k \frac{m}{r}>0$, and (4) holds for $x \in G_{3} \backslash X$.

Let now $x \in S \backslash G_{3}$. Then we may assume that

$g_{1}(x), \ldots, g_{k}(x) \geq 0, \quad 0>g_{k+1}(x), \ldots, g_{l}(x) \geq-\delta, \quad g_{l+1}(x), \ldots, g_{r}(x)<-\delta$, where $0 \leq k \leq l<r$. Then

$$
g_{i}(x) \cdot \varphi\left(g_{i}(x)\right)<\frac{m}{r+1} \text { for } i=1, \ldots, k,
$$

and

$$
g_{i}(x) \cdot \varphi\left(g_{i}(x)\right)<0 \text { for } i=k+1, \ldots, l .
$$

Since $\varphi(t)>B$ for $t \in[-A,-\delta]$, we see that

$g_{i}(x) \cdot \varphi\left(g_{i}(x)\right)<-\delta B=A(-M-r \varepsilon)=-A M-\frac{r m}{r+1} \quad$ for $i=l+1, \ldots, r$.

Hence,

$$
g_{i}(x) \cdot \varphi\left(g_{i}(x)\right)<-M-\frac{r m}{r+1} \quad \text { for } i=l+1, \ldots, r
$$

since $A \geq 1$.

Therefore,

$$
f(x)-\sum_{i=1}^{r} g_{i}(x) \cdot \varphi\left(g_{i}(x)\right)>-M-k \frac{m}{r+1}+(r-l)\left(M+\frac{r m}{r+1}\right)>0 .
$$

This ends the proof of Lemma 1.

Let $a, b \in \mathbb{R}$ and $N \in \mathbb{N}$ be an even number such that for $\varphi(t)=(a t+b)^{N}$ the inequality (4) holds. Let $\operatorname{deg} g_{i}=d_{i}$ for $i=1, \ldots, r$, and let

$$
D_{0}:=\max \left\{d,(N+1) d_{1}, \ldots,(N+1) d_{r}\right\} .
$$

Recall that $d=\operatorname{deg} f$ is an even number. Therefore $D_{0}$ is an even number too. Set

$$
\alpha_{i}=D_{0}-(N+1) d_{i} \text { for } i=1, \ldots, r .
$$

Obviously the $\alpha_{i}$ are nonnegative even numbers.

Lemma 2. The function $F: \mathbb{R}^{n} \rightarrow \mathbb{R}$ defined by

$$
F(x)=|x|^{D_{0}-d} f(x)-\sum_{i=1}^{r}|x|^{\alpha_{i}} g_{i}(x)\left(a g_{i}(x)+b|x|^{d_{i}}\right)^{N}
$$

is a homogeneous polynomial of degree $D_{0}$. 
Proof. We show that $F(t x)=t^{D_{0}} F(x)$. Indeed,

$$
F(t x)=|t x|^{D_{0}-d} f(t x)-\sum_{i=1}^{r}|t x|^{\alpha_{i}} g_{i}(t x)\left(a g_{i}(t x)+b|t x|^{d_{i}}\right)^{N} .
$$

Since the $\alpha_{i}$ are even numbers and the polynomials $f, g_{1}, \ldots, g_{r}$ are homogeneous, the right hand side of (12) is equal to

$$
t^{D_{0}}|x|^{D_{0}-d} f(x)-\sum_{i=1}^{r} t^{\alpha_{i}+d_{i}+N d_{i}}|x|^{\alpha_{i}} g_{i}(x)\left(a g_{i}(x)+b|x|^{d_{i}}\right)^{2 N} .
$$

Hence, by the definition of the $\alpha_{i}$, we deduce the assertion.

Let $F \in \mathbb{R}[x]$ be the polynomial defined by (11).

Lemma 3. For any $x \in \mathbb{R}^{n}, x \neq 0$, we have

$$
F(x)>0 \text {. }
$$

Proof. If $x \neq 0$, then $x=t x_{0}$ for some $x_{0} \in S$ and $t>0$. Hence, $F(x)=$ $t^{D_{0}} F\left(x_{0}\right)$ and by Lemma 1 we have $f\left(x_{0}\right)-\sum_{i=1}^{r} g_{i}\left(x_{0}\right) \varphi\left(g_{i}\left(x_{0}\right)\right)>0$. Thus $F(x)>0$.

To sum up, we have shown that the polynomial $F$ is homogeneous and it is positive for $x \in \mathbb{R}^{n} \backslash\{0\}$, so we can use the Reznick theorem. Therefore we can represent $|x|^{2 r} F$, for some $r \in \mathbb{N}$, as a sum of even powers of linear polynomials.

Now we prove Theorem 3.

Proof of Theorem 3. Take a polynomial of the form (11) such that the assertion of Lemma 2 holds. By Lemma 3,

$$
F(x)>0 \text { for } x \in \mathbb{R}^{n} \backslash\{0\} .
$$

Take $\epsilon(F)=\frac{\inf \{F(u): u \in S\}}{\sup \{F(u): u \in S\}}$, and let $r \in \mathbb{N}$ be such that

$$
r \geq \frac{n D_{0}\left(D_{0}-1\right)}{(4 \log 2) \epsilon(F)}-\frac{n+D_{0}}{2} .
$$

So, for $D=D_{0}+2 r$, by Theorem 1 there exist linear functions $q_{1}, \ldots, q_{j} \in \mathbb{R}[x]$ such that

$$
|x|^{2 r} F(x)=q_{1}^{D}+\cdots+q_{j}^{D} .
$$

Hence

$$
|x|^{D-d} f(x)-\sum_{i=1}^{r}|x|^{\alpha_{i}+2 r} g_{i}(x)\left(a g_{i}(x)+b|x|^{d_{i}}\right)^{N}=\sum_{i=1}^{j} q_{i}^{D}
$$

and

$$
f(x)=|x|^{-D+d}\left(\sum_{i=1}^{j} q_{i}^{D}+\sum_{i=1}^{r}|x|^{\alpha_{i}+2 r} g_{i}(x)\left(a g_{i}(x)+b|x|^{d_{i}}\right)^{N}\right),
$$

which completes the proof of Theorem 3 . 
Open Access. This article is distributed under the terms of the Creative Commons Attribution 4.0 International License (http://creativecommons.org/licenses/ by/4.0/), which permits unrestricted use, distribution, and reproduction in any medium, provided you give appropriate credit to the original author(s) and the source, provide a link to the Creative Commons license, and indicate if changes were made.

\section{References}

[1] E. Artin, Über die Zerlegung definiter Functionen in Quadrate, Abh. Math. Sem. Univ. Hamburg 5 (1927), 100-115; Collected Papers, 273-288, AddisonWesley, Reading, MA, 1965.

[2] T. JACOBI, A representation theorem for certain partially ordered commutative rings, Math. Z. 237 (2001), 259-273.

[3] T. Jacobi And A. Prestel, Distinguished representations of strictly positive polynomials, J. Reine Angew. Math. 532 (2001), 223-235.

[4] J.-L. Krivine, Anneaux préordonnés. J. Analyse Math. 12 (1964), 307-326.

[5] K. Kurdyka And S. Spodzieja, Convexifying positive polynomials and sums of squares approximation, arXiv:1507.06191 (2015)

[6] J. B. LASserRe, Representation of nonnegative convex polynomials, Arch. Math. 91 (2008), 126-130.

[7] M. Putinar, Positive polynomials on compact semi-algebraic sets, Indiana Univ. Math. J. 42 (1993), 969-984.

[8] M. Putinar and F.-H. Vasilescu, Positive polynomials on semi-algebraic sets, C. R. Acad. Sci. Paris Sér. I Math. 328 (1999), 585-589.

[9] M. Putinar and F.-H. Vasilescu, Solving moment problems by dimensional extension, Ann. of Math. 149 (1999), 1087-1107.

[10] B. Reznick, Uniform denominators in Hilbert's seventeenth problem, Math. Z. 220 (1995), 75-97.

[11] C. Scheiderer, A Positivstellensatz for projective real varieties, Manuscripta Math. 138 (2012), 73-88.

[12] K. Schmüdgen, The $K$-moment problem for compact semialgebraic sets, Math. Ann. 289 (1991), 203-206.

[13] M. Schweighofer, Iterated rings of bounded elements and generalizations of Schmüdgen's Positivstellensatz. J. Reine Angew. Math. 554 (2003), 19-45.

[14] M. Schweighofer, Optimization of polynomials on compact semialgebraic sets, SIAM J. Optim. 15 (2005), 805-825 (electronic).

[15] S. Spodzieja, A geometric model of an arbitrary real closed field, Pacific J. Math. 264 (2013), 455-469.

[16] G. Stengle, A Nullstellensatz and a Positivstellensatz in semialgebraic geometry, Math. Ann. 207 (1974), 87-97. 


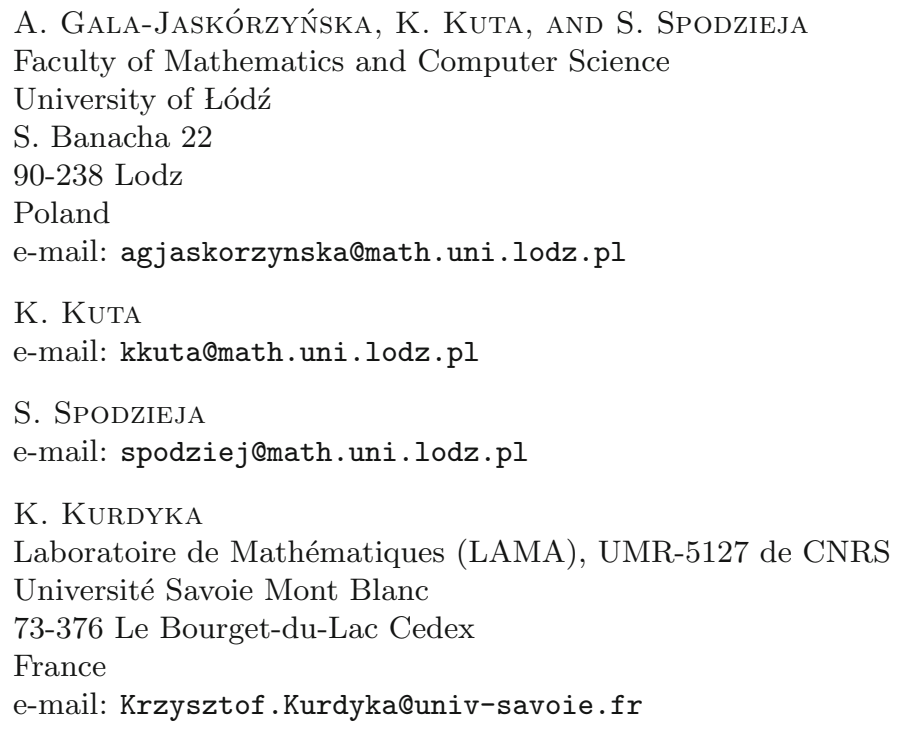

Received: 28 April 2015 